\title{
Coeficiente de Digestibilidad Aparente de Plantas Forrajeras Comunes en Zona Andina para Alimentación de Tilapia Nilótica (Oreochromis niloticus)
}

\author{
Christine M. Hahn-von-Hessberg(1), Alberto Grajales-Quintero(1) y William Narváez-Solarte ${ }^{(2)}$. \\ Universidad de Caldas, Facultad de Ciencias Agropecuarias, (1) Depto. Producción Agropecuaria, \\ (2) Depto. Salud Animal, Calle 65 No. 26-10, Manizales, Caldas-Colombia. \\ (e-mail: christine.hahn@ucaldas.edu.co, alberto.grajales@ucaldas.edu.co; wnarvaez@ucaldas.edu.co)
}

Recibido Oct. 29, 2015; Aceptado Ene. 5, 2016; Versión final Feb. 15, 2016, Publicado Ago. 2016

\begin{abstract}
Resumen
Se evalúan los coeficientes de digestibilidad de la proteína bruta, extracto etéreo, fibra detergente neutro, fibra detergente ácido, calcio, fósforo, extracto libre de nitrógeno y energía bruta de la harina del parénquima de la hoja de: Tithonia diversifolia, Xanthosoma sagittifolium, Trichanthera gigantea y Gliricidia sepium, suministradas a Oreochromis niloticus. Los coeficientes de digestibilidad fueron calculados por el método indirecto, sustituyendo el $30 \%$ de la dieta referencia por el material vegetal a analizar, usando el $\mathrm{Cr}_{2} \mathrm{O}_{3}$ al $0.5 \%$.y distribuyendo los tratamientos en un diseño en bloques completos al azar. Los resultados obtenidos para los diferentes rangos de coeficientes de digestibilidad para la proteína bruta fueron de $89.94 \%$ - $98.66 \%$; extracto etéreo $87.73 \%-98.79 \%$; fibra detergente neutro $17.60 \%$ - $87.73 \%$; calcio $50.44 \%$ - 95.41\%; fósforo $72.21 \%$ - $93.75 \%$; extracto libre de nitrógeno $60.20 \%$ $96.57 \%$; energía bruta $57.31 \%-78.47 \%$. Se concluye que Oreochromis digiere de manera eficiente las plantas forrajeras, convirtiéndose en ingredientes suplementarios alternativos para su alimentación en sistemas agrícolas.
\end{abstract}

Palabras clave:coeficiente de digestibilidad; Tithonia diversifolia;Xanthosoma sagittifolium; Trichanthera gigantea; Gliricidia sepium; Cichlidae; nutrición

\section{Apparent Digestibility Coefficient of Common Fodder Plants in the Andean Region for the Feeding of Nile Tilapia (Oreochromis niloticus)}

\begin{abstract}
Digestibility coefficients of crude protein, ether extract, neutral detergent fiber, acid detergent fiber, calcium, phosphorus, nitrogen free extract and gross energy are evaluated in: Tithonia diversifolia, Xanthosoma sagittifolium, Trichanthera gigantea and Gliricidia sepium, supplied to Oreochromis niloticus. The digestibility coefficients were calculated using the indirect method, replacing $30 \%$ of the reference diet by the plant material to be analyzed, using the $0.5 \% \quad \mathrm{Cr}_{2} \mathrm{O}_{3}$, and distributing the treatments in a randomized block design. The results obtained for different ranges of digestibility coefficients for crude protein were $89.94 \%$ - 98.66\%; ether extract $87.73 \%$ - 98.79\%; neutral detergent fiber $17.60 \%-87.73 \%$; Calcium 50.44\% - 95.41\%; phosphorus $72.21 \%$ - 93.75\%; nitrogen-free extract $60.20 \%$ - $96.57 \%$; gross energy $57.31 \%-78.47 \%$. In conclusion, Oreochromis efficiently digested forage plants, becoming an alternative supplemental ingredient for food in farming systems.
\end{abstract}

Keywords: digestibility coefficient; Tithonia diversifolia;Xanthosoma sagittifolium; Trichanthera gigantea; Gliricidia sepium; Cichlidae; nutrition 


\section{INTRODUCCIÓN}

En la producción pecuaria, la alimentación representa el $73 \%$ de los costos operativos. En piscicultura se adicionan materias primas de alto costo, como la harina de pescado, lo cual genera la búsqueda de alternativas de menor precio, fácilmente asequibles y de bajo impacto ambiental. Conocer el coeficiente de digestibilidad (CD) de los ingredientes de una dieta para peces, da el soporte para evaluar y obtener una dieta de óptima calidad. La digestibilidad aparente de un componente varía dependiendo de su procedencia vegetal o animal, procesamiento, interacción con los demás ingredientes, métodos de análisis, factores ambientales y especie íctica a la que se le suministra, donde, no se tienen en cuenta las partículas provenientes de enzimas, células epiteliales y microbiales (Secombe y Lester, 2012). En peces el método más utilizado para determinar el coeficiente de digestibilidad aparente de las dietas, es recolectando las heces para su análisis, que puede ser por método directo (in vivo) o por extracción directa de la materia fecal por stripping (Rodríguez et al., 2007). Las sustancias más utilizadas como indicadoras inertes son el óxido de cromo III $\left(\mathrm{Cr}_{2} \mathrm{O}_{3}\right)$ y el dióxido de titanio $\left(\mathrm{TiO}_{2}\right)$, estos no deben ser asimilados, ni tener efecto en la digestión o causar efectos fisiológicos secundarios, ser fácilmente medible y excretado (Kavanagh et al., 2001).

Se han realizado distintos trabajos en la búsqueda de materias primas no convencionales para peces de cultivo como oleaginosas, plantas acuáticas, proteínas unicelulares, leguminosas y subproductos de cereales, entre otros. Así la torta de soya sustituyó a la harina de pescado en un $25 \%$ y la cañafístula (Moringa oleífera) puede llegar a ofrecerse del 10 al 30\% de la proteína de la ración (Rivas-Vega et al., 2012). La digestibilidad de la proteína de las hojas de maní (Arachis hypogaea) es del $80-83 \%$ (GarduñoLugo y Olvera-Novoa, 2008), lenteja de agua (Lemna sp.) del 79\% (Nhi y Preston, 2012), y la harina de fríjol serpiente (Vigna unguiculata) del 68\% (El-Saidy y Saad, 2008), la harina de alfalfa (Medicago sativa) permite una sustitución del $35 \%$ de la proteína animal, y la harina de hoja de acacia (Leucaena leucocephala).

El botón de oro (Thitonia diversifolia) (Hemsl.) A. Gray, Familia Compositae, se adapta al clima tropical húmedo y seco entre los 0 a $2500 \mathrm{msnm}$, se reconoce por su elevado contenido de nutrientes en materia seca, ingrediente potencial en la dieta de diferentes producciones pecuarias (González-Castillo et al., 2014). Esta planta no compite con la alimentación humana poco se conoce sobre los coeficientes de digestibilidad de sus nutrientes en peces. Según Verdecia et al. (2011), el corte óptimo del material vegetal del botón de oro, debe realizarse entre los 30 a 40 días, donde la PB en base seca varía del 18,9\% al 28,75\%, FB entre $1,63 \%$ y $3,83 \%$, materia seca del $23 \%$, ceniza de 21,4 al $78,6 \%$ en materia orgánica, FDN $34,4 \%$ a $50,5 \%$ y FDA de 27,7 a $32,1 \%$.

En la literatura consultada no hay claridad sobre la identificación de los géneros Xanthosoma y Alocasia para la planta comúnmente denominada Bore. El Bore de la Estación Piscícola de la Universidad de Caldas, fue clasificada como Xanthosoma sagittifolium (L.) Schott por el Missouri Botanical Garden (U.S.A.). Pertenece a la Familia Araceae, crece entre 16 a $25^{\circ} \mathrm{C}$ de temperatura, inicia su producción entre los cuatro a seis meses, con cosechas cada 40 días promedio, sus hojas en materia seca presentan una proteína bruta entre $14 \%$ y $22 \%$ (Gómez, et al., 2002). En base fresca, el Bore se evaluó con Tilapia rendalli, en la Universidad de Caldas, para programas de piscicultura campesina. Con el suministro de follaje fresco de Bore a la tilapia nilótica se obtuvo una ganancia promedio de $0,36 \mathrm{~g} / \mathrm{pez} /$ día y una conversión alimenticia de 2,42 (Hahn et al., 2002).

El Nacedero (Trichanthera gigantea) (Bonpl.) Nees, Familia Acanthaceae, se adapta a clima tropical húmedo y seco, crece entre los 0 a $2100 \mathrm{msnm}$, usado como alimento alternativo para diferentes especies de animales. Según Brenes-Soto (2014) presenta una MS del 12,4\%, PC 25\%, EE 1,77\%, P 0,28\%, Ca $5,36 \%$, sugiere reemplazar entre el $20 \%$ al $30 \%$ del aporte proteico de la proteína de las dietas para cerdas y conejos. El Matarratón (Gliricidia sepium) (Jacq.), Kunth ex Walp, Familia Fabaceae, de uso frecuente con otros cultivos o como cerca viva y sombrío. Presenta en base seca en promedio $23 \%$ de proteína bruta, $45 \%$ fibra bruta, $1,7 \%$ de calcio y $0,2 \%$ de fósforo (Gómez et al., 2002). Ha presentado un buen desempeño en conejos, bovinos, ovinos, caprinos y curíes, obteniendo para ésta última especie un CD de la fracción protéica del 91,6\% (Narváez et al., 2012).

No obstante, la alta calidad de estas plantas forrajeras y los estudios en las diversas especies animales, la investigación en la alimentación de peces es escasa, razón por la cual el objetivo de este trabajo es determinar los coeficientes de digestibilidad aparente (CDA) de la proteína bruta (PB), extracto etéreo (EE), fibra en detergente neutro (FDN), fibra en detergente ácido (FDA), calcio (Ca), fósforo (P), extracto libre de nitrógeno (ELN) y de energía bruta (EB) obtenidos de la harina del parénquima de la hoja de botón de oro, bore, nacedero y matarratón para ser incluida en la alimentación de la tilapia en fase de crecimiento, buscando alternativas, en especial, para programas de seguridad alimentaria. 


\section{MATERIALES Y MÉTODOS}

Se realizó el bioensayo en el Laboratorio Ictiológico de la Estación Piscícola de la Universidad de Caldas, ubicada en el municipio de Palestina del departamento de Caldas - Colombia, a $75^{\circ} 45^{\prime}$ de Longitud Occidental y $5^{\circ} 04^{\prime}$ de Latitud Norte, $1.050 \mathrm{msnm}$, humedad relativa de $75 \%$, precipitación anual de 2.377 $\mathrm{mm}$, temperatura ambiental promedio de $22,5^{\circ} \mathrm{C}$ y brillo solar anual de 2049 horas.

Para la aclimatación y alimentación previa, el lote de peces se alojó en tanques de concreto en el laboratorio ictiológico a una densidad de tres animales por metro cuadrado. Para el experimento se utilizaron tanques de fibra de vidrio de fondo cónico con capacidad de 250 litros de agua cada uno, provistos al final con un recipiente de $350 \mathrm{ml}$ para la decantación y recolección de las heces. El laboratorio cuenta con un sistema de recirculación de agua y oxigenación constante, garantizando condiciones físico-químicas del agua estables, los parámetros del agua fueron monitoreados con un medidor de $\mathrm{pH}$, temperatura y oxígeno disuelto (marca Dakton de Luxe ph/disolved oxygen meter kit), manteniendo rangos óptimos para la especie. Se utilizaron ejemplares machos de tilapia nilótica, llevados a reversión sexual y levante. Se emplearon diez peces por cada unidad experimental, con peso promedio de 100 \pm 10 gramos, distribuidos al azar para un total de 40 ejemplares para cada uno de los materiales vegetales (MV) estudiados, colocados en tanques de concreto hasta su traslado a los tanques de fibra de vidrio con fondo cónico dentro de jaulas malla plástica de 17x17 $\mathrm{mm}$ para evitar el estrés ocasionado por la manipulación y permitiendo el paso de la materia fecal al recipiente de recolección en la parte inferior.

El alimento suministrado a los peces consta de una dieta referencia semipurificada (DR) (Tabla 1), la cual satisface los requerimientos nutricionales de la tilapia nilótica en la fase de crecimiento (NRC, 1993), con un $30 \%$ de proteína, empleando la metodología descrita por Vásquez-Torres et al. (2010), se utilizaron ingredientes altamente digestibles y libres de factores anti-nutricionales (Tabla 1), como indicador inerte se usó el óxido de cromo III $\left(\mathrm{Cr}_{2} \mathrm{O}_{3}\right)$, las hojas de los MV fueron cosechadas en promedio a los 40 días de edad, secadas en condiciones de invernadero, con temperatura promedio de $60^{\circ} \mathrm{C}$ hasta obtener una humedad inferior al $8 \%$.

Tabla 1. Composición porcentual de la dieta referencia (DR) (base húmeda) según Vásquez-Torres et al. (2010).

\begin{tabular}{|l|c|}
\hline Ingredientes & (\%) \\
\hline Caseina $^{1}$ & 33,3 \\
\hline Gelatina $^{2}$ & 3,4 \\
\hline Dextrina & 40,0 \\
\hline Alfa-Celulosa & 13,6 \\
\hline Aceite de pescado & 2,4 \\
\hline Aceite de girasol & 2,4 \\
\hline Premezcla de Vitaminas $^{3}$ & 0,2 \\
\hline Premezcla de Microminerales $^{4}$ & 0,1 \\
\hline Premezcla de Macrominerales $^{5}$ & 4,0 \\
\hline Ácido ascórbico & 0,1 \\
\hline Óxido de cromo III $_{\left(\mathrm{Cr}_{2} \mathrm{O}_{3}\right)}$ & 0,5 \\
\hline Total & 100 \\
\hline
\end{tabular}

La composición de los ingredientes utilizados fue: 1. Caseina: MS 93,5\%, PB 85,5\%, lípidos 2,4\%, cenizas 3,5\%; 2. Gelatina: MS 94\%; PB 95\%; 3. Premezcla Vitaminas: Vit A 7.9*106 UI, Vit $D_{3}, 1.8^{\star} 106$ UI, Vit E $68 \mathrm{~g}$ Vit $B_{1} 6.7 \mathrm{~g}$, Vit $B_{2} 13.4 \mathrm{~g}$, Vit $B_{5} 50.000 \mathrm{mg}$, Vit $B_{6} 6.66 \mathrm{~g}$, Pantotenato de Ca $33 \mathrm{~g}$, Biotina $500 \mathrm{mg}$, Ac. Fólico $5.000 \mathrm{mg}$, Ac. Ascórbico $250.000 \mathrm{mg}$, Ac. Nicotínico $99.97 \mathrm{~g}$, Vit B $1220 \mathrm{mg}$, Vit K $6.8 \mathrm{~g}$, Niacina $45.000 \mathrm{mg}$ vehículo csp. $1.0 \mathrm{~kg}$; 4. Premezcla Microminerales: Composición por $100 \mathrm{~g}$ : Magnesio 1.0, Zinc $130.000 \mathrm{mg}$, Hierro $170.000 \mathrm{mg}$, Cobre $15.000 \mathrm{mg}$, lodo $2.000 \mathrm{mg}$, Selenio $300 \mathrm{mg}$. Cobalto 0.02 , Manganeso $15.000 \mathrm{mg}$; 5 . Premezcla de Macrominerales: Composición por $100 \mathrm{~g}$ : $\mathrm{Ca}\left(\mathrm{H}_{2} \mathrm{PO}_{4}\right) 12$ g; Lactato de Ca 33.33g; 2MgSO 4.65; $\mathrm{H}_{2} \mathrm{O}, 12.5 \mathrm{~g} ; \mathrm{KH}_{2} \mathrm{PO}_{4} 23.5 \mathrm{~g} ; \mathrm{NaCl} 4.5 \mathrm{~g} ; \mathrm{AlCl}_{3} 0.02 \mathrm{~g}, \mathrm{CMC}_{2} 9.5 \mathrm{~g}$; y 6 . Ácido ascórbico: Stay-C-50

La composición química de la DR y de las cuatro plantas forrajeras en base seca a evaluar, se encuentran, respectivamente en las Tablas 1 y 2 . El parénquima de las hojas secas de las plantas forrajeras se molieron a un diámetro de $300 \mu \mathrm{m}$ y se almacenaron en bolsas plásticas a una temperatura de $-^{\circ}{ }^{\circ} \mathrm{C}$. Para la elaboración de la DR (Tabla 1), y de la dieta experimental (DE), se mezclaron los ingredientes hasta su 
homogenización, adicionando agua a $24^{\circ} \mathrm{C}$, al $20 \%$ del peso total de la dieta, se utilizó un equipo manual de tornillo sinfín, obteniéndose pellets de $4,0 \mathrm{~mm}$ de diámetro. La DR fue utilizada para el acostumbramiento inicial de los peces, posteriormente, se realizaron las DE, donde se empleó, un $69,5 \%$ de la DR, adicionándole un $30 \%$ de la harina del parénquima de la hoja de la planta forrajera a evaluar y el $0,5 \%$ de óxido de cromo III, los pellets obtenidos en ambas dietas (DR y DE) se secaron a $60^{\circ} \mathrm{C}$ durante 4 horas promedio y se almacenaron en bolsas plásticas a $-4^{\circ} \mathrm{C}$, hasta su uso.

Para la preparación de los ejemplares, se considera que el tiempo de vaciamiento del tracto digestivo y adaptación a la DE es suficiente con 15 días de anticipación (Giraldo et al., 2008), con un período de acostumbramiento de cinco días, donde los peces recibieron únicamente la DR, posteriormente y durante diez días consecutivos, fueron alimentados con las dietas experimentales que contenían la harina del parénquima de las hojas del MV a evaluar, en ambos casos se les suministró el alimento en tres comidas diarias, a las nueve, trece y quince horas de cada día hasta la saciedad. Al quinceavo día, después de la segunda comida, los animales se trasladaron a los tanques cónicos para la recolección de heces, donde a partir de la octava hora, se comenzó a recolectar las heces por decantación en el dispositivo removible, a intervalos de una hora durante 18 horas.

Los ejemplares se devolvieron al respectivo tanque de alimentación para su recuperación durante quince dias, tiempo en el cual recibieron la DR; posteriormente, y por diez días se les suministró la nueva dieta experimental. Las heces recolectadas fueron secadas a $60^{\circ} \mathrm{C}$, maceradas en mortero, homogenizadas, empacadas en bolsas plásticas y almacenadas a $-4^{\circ} \mathrm{C}$ hasta su análisis. El uso del método Guelph modificado, permite que los peces se alimenten independientemente al sistema empleado para la colecta de heces, evitando la contaminación del material recolectado por la respectiva dieta. Los análisis químicobromatológicos de los MV, dietas, heces y la concentración de óxido de cromo III, fueron realizados en un laboratorio particular certificado por el Instituto Colombiano Agropecuario, según los procedimientos recomendados por la AOAC (1995). El análisis de energía se realizó en Isoperibol calorimeter 6200 (Parr Instrument Company) en el Laboratorio de Nutrición Animal y Vegetal de la Universidad de Caldas. El coeficiente de digestibilidad aparente (CDA) fue calculado usando la siguiente ecuación:

$\mathrm{Da}(\mathrm{n})=100-\left[100\left(\% \mathrm{Cr}_{2} \mathrm{O}_{3 r} /\left(\% \mathrm{Cr}_{2} \mathrm{O}_{3 \mathrm{f}}\right) \times\left(\% \mathrm{~N}_{\mathrm{f}} / \% \mathrm{~N}_{\mathrm{r}}\right)\right]\right.$

$\mathrm{Da}(\mathrm{n})=\left[100\left(\% \mathrm{Cr}_{2} \mathrm{O}_{3}\right)\right]$

Donde, (1-2) $\mathrm{Da}(\mathrm{n})=$ Digestibilidad aparente; $\mathrm{Cr}_{2} \mathrm{O}_{3} \mathrm{r}=\%$ de óxido de cromo-III en la ración; $\mathrm{Cr}_{2} \mathrm{O}_{3} \mathrm{f}=\%$ de óxido de cromo-III fecal; $\mathrm{Nr}$ = Nutriente en la ración; $\mathrm{Nf}=$ Nutriente en las heces.

El coeficiente de la digestibilidad aparente de los nutrientes de cada alimento fue calculado de acuerdo a la siguiente ecuación, propuesta por Forster (1999):

$\operatorname{CDAN}_{\mathrm{mv} .}=\left[(\mathrm{a}+\mathrm{b}) \times \mathrm{CDAN}_{\mathrm{exp}}-\mathrm{a} \times \mathrm{CDAN}_{\text {ref. }}\right] / \mathrm{b}$

Donde, (3) $\mathrm{CDAN}_{\mathrm{mv}}=$ coeficiente de digestibilidad aparente del nutriente en el material vegetal; $C D A N_{\exp }=$ coeficiente de digestibilidad aparente del nutriente en la dieta experimental; $C D A N_{\text {ref }}=$ coeficiente de digestibilidad aparente del nutriente en la dieta referencia; $a=$ contribución del nutriente de la dieta referencia al contenido de nutriente de la dieta sustituida; $b=$ contribución del nutriente del material vegetal a evaluar en el contenido de nutriente de la dieta sustituida; $a+b=$ nivel de nutriente de la dieta sustituida.

Se tomaron muestras del parénquima de la hoja de los MV en materia natural para determinar la humedad y MS de los mismos, y establecer la concentración de nutrientes digestibles en base fresca. Para obtener los CD de las dietas experimentales y de los nutrientes de los MV, se realizó el análisis bromatológico de la harina del parénquima de las hojas, de las dietas experimentales, de la dieta referencia y de las heces respectivas de cada dieta, para determinar proteína bruta $(\mathrm{PB})$, extracto etéreo $(\mathrm{EE})$, cenizas, calcio $(\mathrm{Ca})$, fósforo $(P)$, extracto libre de nitrógeno (ELN) y energía bruta (EB) según la metodología de la AOAC (1995), la fibra bruta (FB), fibra en detergente neutro (FDN) y la fibra en detergente ácido (FDA) se aplicó la metodología de Van Soest (1991). El diseño experimental se constituyó de cuatro tratamientos experimentales (cuatro MV) y una DR, con cuatro repeticiones cada uno, distribuidos en un diseño de bloques completos al azar y distribuidos en el tiempo. Los resultados fueron sometidos al análisis de varianza de una sola vía. En el caso de encontrar diferencia estadística significativa $(p<0,05)$, entre las medias de tratamientos, se utilizó la prueba de comparación de medias de Tukey $(p<0,05)$. Los datos fueron analizados utilizando el programa estadístico SAS (2007). 


\section{RESULTADOS}

Los valores medios obtenidos para temperatura, oxígeno y $\mathrm{Ph}$, permanecieron dentro de los rangos recomendados para la especie estudiada, así la temperatura se conservó entre $23,4 \pm 1^{\circ} \mathrm{C}$, nivel de oxígeno

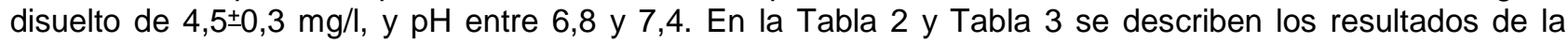
composición bromatológica y contenido de energía bruta en base seca y base fresca, respectivamente, de las plantas forrajeras investigadas.

Tabla 2. Composición bromatológica y contenido de energía bruta en base seca del botón de oro (HBO), bore (HB), nacedero $(\mathrm{HN})$ y matarratón $(\mathrm{HM})$.

\begin{tabular}{|c|c|c|c|c|c|c|c|c|c|c|}
\hline Material Vegetal & $\begin{array}{c}P B \\
\%\end{array}$ & $\begin{array}{c}E E \\
\%\end{array}$ & $\begin{array}{c}\text { Cenizas } \\
\%\end{array}$ & $\begin{array}{c}P \\
\%\end{array}$ & $\begin{array}{c}C a \\
\%\end{array}$ & $\begin{array}{c}F B \\
\%\end{array}$ & $\begin{array}{c}F D N \\
\%\end{array}$ & $\begin{array}{c}F D A \\
\%\end{array}$ & $\begin{array}{c}E L N \\
\%\end{array}$ & $\begin{array}{c}E B \\
K c a l / k g\end{array}$ \\
\hline HBO & 32,0 & 1,01 & 10,88 & 0,39 & 2,71 & 7,73 & 49,23 & 8,06 & 53,42 & 4145,81 \\
\hline HB & 23,5 & 4,47 & 24,40 & 0,31 & 1,98 & 9,36 & 48,71 & 7,73 & 44,46 & 4348,77 \\
\hline HN & 27,0 & 1,72 & 14.07 & 0,23 & 1,73 & 8,06 & 55,83 & 4,06 & 44,86 & 3737,04 \\
\hline HM & 20,5 & 0,89 & 8,77 & 0,27 & 7,01 & 4,06 & 59,23 & 9,34 & 58,45 & 3112,51 \\
\hline
\end{tabular}

Tabla 3. Composición bromatológica y contenido de energía bruta en base fresca del botón de oro (HBO), bore (HB), nacedero $(\mathrm{HN})$ y matarratón $(\mathrm{HM})$.

\begin{tabular}{|c|c|c|c|c|c|c|c|c|c|c|c|}
\hline $\begin{array}{c}\text { Material } \\
\text { Vegetal }\end{array}$ & $\begin{array}{c}M S \\
\%\end{array}$ & $\begin{array}{c}P B \\
\%\end{array}$ & $\begin{array}{c}E E \\
\%\end{array}$ & $\begin{array}{c}\text { Cenizas } \\
\%\end{array}$ & $\begin{array}{c}P \\
\%\end{array}$ & $\begin{array}{c}C a \\
\%\end{array}$ & $\begin{array}{c}F B \\
\%\end{array}$ & $\begin{array}{c}F D N \\
\%\end{array}$ & $\begin{array}{c}F D A \\
\%\end{array}$ & $\begin{array}{c}E L N \\
\%\end{array}$ & $\begin{array}{c}E B \\
K c a l / k g\end{array}$ \\
\hline HBO & 15,71 & 5,03 & 0,16 & 1,71 & 0,06 & 0,43 & 1,21 & 7,73 & 1,27 & 8,39 & 651,17 \\
\hline HB & 20,53 & 4,82 & 0,92 & 5,00 & 0,06 & 0,41 & 1,92 & 10,00 & 1,59 & 9,12 & 892.64 \\
\hline HN & 10,61 & 2,86 & 0,18 & 1,49 & 0,02 & 0,18 & 0,85 & 5,92 & 0,43 & 4,75 & 396,49 \\
\hline HM & 20,62 & 4,23 & 0,18 & 1,81 & 0,06 & 1,45 & 0,83 & 12,21 & 1,93 & 12,05 & 641,69 \\
\hline
\end{tabular}

Los resultados de la composición bromatológica en base seca demuestran que el mayor porcentaje de PB y fósforo total lo presenta la HBO con un $32 \%$ y $0,39 \%$, respectivamente, y se corresponde con el mayor contenido de estos nutrientes en base fresca con valores 5,03\% y $0,06 \%$, respectivamente. La HB presentó, tanto en base seca como en base fresca, los mayores contenidos de extracto etéreo, cenizas, fibra bruta y energía bruta. La HN se caracteriza por presentar el mayor contenido de humedad en base fresca con un valor de $89,39 \%$, lo que repercute en la baja concentración de los nutrientes. Finalmente, la HM aunque presentó el mayor contenido de materia seca en base fresca, fue el MV que presentó los menores contenidos de PB y EB en base seca con valores de 20,5 y $3112,51 \mathrm{kcal} / \mathrm{kg}$, respectivamente. En la Tabla 4 se presentan los Coeficientes de Digestibilidad Aparente (CDA) de los nutrientes contenidos en los materiales vegetales estudiados.

Tabla 4: Coeficientes de Digestibilidad Aparente (CDA) de los nutrientes del botón de oro (HBO), bore (HB), nacedero $(\mathrm{HN})$ y matarratón (HM) en la alimentación de tilapia nilótica en fase de crecimiento.

\begin{tabular}{|c|c|c|c|c|c|c|c|c|}
\hline $\begin{array}{c}\text { Material } \\
\text { Vegetal }\end{array}$ & CDA PB\% & CDA EE\% & $\begin{array}{c}\text { CDA } \\
\text { P\% }\end{array}$ & CDA Ca\% & $\begin{array}{c}\text { CDA } \\
\text { FDN\% }\end{array}$ & $\begin{array}{c}\text { CDA } \\
\text { FDA\% }\end{array}$ & $\begin{array}{c}\text { CDA } \\
\text { ELN\% }\end{array}$ & CDA EB\% \\
\hline HBO & $91,3 \pm 1,1 \mathrm{~b}$ & $98,7 \pm 1,5 \mathrm{a}$ & $93,7 \pm 2,0 \mathrm{a}$ & $95,4 \pm 1,5 \mathrm{a}$ & $77,9 \pm 1,8 \mathrm{~b}$ & $54,0 \pm 2,5 \mathrm{~b}$ & $60,2 \pm 3,4 \mathrm{c}$ & $57,3 \pm 3,0 \mathrm{~b}$ \\
\hline $\mathrm{HB}$ & $89,9 \pm 2,0 \mathrm{c}$ & $92,2 \pm 1,4 \mathrm{~b}$ & $72,2 \pm 1,8 \mathrm{~b}$ & $50,4 \pm 2,1 \mathrm{~b}$ & $17,6 \pm 2,7 \mathrm{c}$ & $18,5 \pm 1,9 \mathrm{~d}$ & $90,9 \pm 3,7 \mathrm{a}$ & $78,4 \pm 2,3 \mathrm{a}$ \\
\hline $\mathrm{HN}$ & $98,6 \pm 1,9 \mathrm{a}$ & $90,7 \pm 2,3 \mathrm{~b}$ & $91,7 \pm 1,9 \mathrm{a}$ & $94,1 \pm 3,0 \mathrm{a}$ & $87,7 \pm 1,6 \mathrm{a}$ & $64,7 \pm 2,7 \mathrm{a}$ & $87,0 \pm 1,0 \mathrm{~b}$ & $73,5 \pm 2,0 \mathrm{a}$ \\
\hline $\mathrm{HM}$ & $93,6 \pm 0,9 \mathrm{~b}$ & $87,7 \pm 2,4 \mathrm{c}$ & $93,6 \pm 2,2 \mathrm{a}$ & $94,4 \pm 2,9 \mathrm{a}$ & $76,3 \pm 0,8 \mathrm{~b}$ & $44,8 \pm 1,5 \mathrm{c}$ & $96,5 \pm 1,7 \mathrm{a}$ & $75,2 \pm 1,3 \mathrm{a}$ \\
\hline Probabilidad & 0,046 & 0,035 & 0,002 & 0,026 & 0,001 & 0,001 & 0,039 & 0,029 \\
\hline
\end{tabular}

Medias de tratamientos dentro de la misma columna, seguidas por letras minúsculas diferentes, son estadísticamente diferentes entre sí $(p<0,05)$ por la prueba de Tukey. Al realizar el análisis de varianza de los coeficientes de digestibilidad se observaron diferencias estadísticas significativas entre medias de tratamientos $(p<0,05)$, en todos los nutrientes evaluados (Tabla 4). Al proceder con la prueba de comparación múltiple de medias de los coeficientes de digestibilidad aparente de la PB, se encontró que la 
HN presentó el mayor valor $(p<0,05)$ seguido de la HBO y HM que son estadísticamente iguales $(p>0,05)$ entre ellos y superiores $(p<0,05)$ al de la HB. Para el CDA del extracto etéreo se presenta el mayor valor $(p$ $<0,05)$ para la HBO, siendo estadísticamente iguales la $\mathrm{HB}$ y la $\mathrm{HN}(p>0,05)$ y superiores a la $\mathrm{HM}$. En los resultados obtenidos para el CDA del fósforo y calcio, la $\mathrm{HBO}, \mathrm{HN}$ y $\mathrm{HM}$ son estadísticamente iguales y presentaron los mejores valores $(p<0,05)$. El valor del CDA de FDN y de FDA de la HN fue estadísticamente superior $(p<0,05)$ al de los demás materiales vegetales, mientras que la $H B$ fue la que presentó el menor CDA $(p<0,05)$ para estos nutrientes. El CDA del Extracto Libre de Nitrógeno de la HB y la HM fueron estadísticamente superiores $(p<0,05)$ a los observados para la HN y la HBO. El coeficiente de digestibilidad aparente para la energía bruta de la HB, HN, HM son estadísticamente similares $(p>0,05)$ $y$ con valor del coeficiente superior $(p<0,05)$ al de la HBO.

Tabla 5. Composición en base fresca del botón de oro ( $\mathrm{HBO})$, bore $(\mathrm{HB})$, nacedero $(\mathrm{HN})$ y matarratón $(\mathrm{HM})$ en nutrientes digestibles y energía digestible para la alimentación de tilapia nilótica en fase de crecimiento.

\begin{tabular}{|c|c|c|c|c|c|c|c|c|}
\hline $\begin{array}{c}\text { Material } \\
\text { Vegetal }\end{array}$ & $\begin{array}{c}\text { Prot. } \\
\text { Digest \% }\end{array}$ & $\begin{array}{c}\mathrm{EE} \\
\text { Digest \% }\end{array}$ & $\begin{array}{c}\mathrm{P} \\
\text { Digest \% }\end{array}$ & $\begin{array}{c}\text { Ca } \\
\text { Digest \% }\end{array}$ & $\begin{array}{c}\text { FDN Digest } \\
\%\end{array}$ & $\begin{array}{c}\text { FDA } \\
\text { Digest \% }\end{array}$ & $\begin{array}{c}\text { ELN } \\
\text { Digest \% }\end{array}$ & $\begin{array}{c}\text { ED } \\
\text { Kcal/kg }\end{array}$ \\
\hline HBO & 4,59 & 0,16 & 0,06 & 0,41 & 6,03 & 0,68 & 5,05 & 373,18 \\
\hline HB & 4,34 & 0,85 & 0,05 & 0,21 & 1,76 & 0,30 & 8,29 & 700,45 \\
\hline HN & 2,83 & 0,17 & 0,02 & 0,17 & 5,20 & 0,28 & 4,13 & 291,50 \\
\hline HM & 3,96 & 0,16 & 0,05 & 1,37 & 9,33 & 0,86 & 11,64 & 482,81 \\
\hline
\end{tabular}

Usando los valores de la composición de los MV en base fresca (Tabla 3) y los respectivos CDA de los nutrientes (Tabla 4), se observó que la HBO fue la de mayor contenido de Proteína y Fósforo digestibles con un $4,59 \%$ y $0,06 \%$ (Tabla 5), respectivamente; mientras que la HM presentó los mayores contenidos de Calcio, FDN, FDA y ELN digestibles y la HB mostró las mayores concentraciones de EE y ED. En los resultados descritos en la Tabla 5 , se destaca la $\mathrm{HN}$ como el MV de menor contenido en nutrientes digestibles e inferior aporte energético para la alimentación de la tilapia nilótica.

\section{DISCUSIÓN}

No se hallaron reportes específicos que permitieran realizar comparaciones de resultados obtenidos con los mismos MV aquí evaluados, por tanto, se utilizó la información existente de materias primas vegetales convencionales de uso frecuente en la alimentación de esta especie, que usualmente son los patrones a ser sustituidos por las no convencionales.

Los MV evaluados en este estudio presentan una PB en base seca del $20,5 \%$ al $32 \%$, con un coeficiente de digestibilidad entre el $89,94 \%$ y $98,66 \%$. Al comparar los datos con los coeficiente de digestibilidad de la proteína de materias primas vegetales, como la torta de soya evaluada en las tilapias, se encuentra que el CDA para la PB varía entre el $85 \%$ y el $92,4 \%$ (Vásques-Torres et al., 2013). Para la harina de soya integral Vásquez-Torres et al. (2010) observó, para el mismo nutriente, CDA entre el 91,9\% al 92,49\% y De Castro et al. (2005) al adicionar enzimas fitasas a esta harina obtuvo un valor de $93,28 \%$. Se han evaluado materias primas alternativas en la alimentación de las tilapias como el fríjol cargamanto (Phaseolus vulgaris), la torta de palmiste (Elaeis guineensis, Jacq), el chachafruto (Erythrina edulis) y la quinua (Chenopodium quinoa), con valores del CDA para la fracción proteica de $84,4 \%, 91,0 \%, 72,4 \%$ y $67 \%$ a $79,9 \%$, respectivamente (Gutiérrez-Espinosa, 2011). Entretanto, al estudiar el CDA de la proteína de materias primas energéticas, caracterizadas por poseer bajo nivel de proteína, como el maíz amarillo (Zea mays), harina de yuca integral (Manihot esculenta) (Vásquez-Torres et al., 2010), sorgo (Sorghum bicolor) y trigo (Triticum sp.) (Sklan et al., 2004), se han obtenido valores de 97,9\%, 89,9\%, 85,5 y 79,5\%, respectivamente.

El National Research Council (1993), afirma que un CD alto de un ingrediente rico en proteína está por encima del $75 \%$, por lo tanto los resultados obtenidos en esta investigación demuestran que la digestibilidad de la PB de los MV estudiados son altos. Según Vásquez-Torres et al. (2010) los alimentos con alta cantidad de MS y bajo coeficiente de digestibilidad, se constituyen en factor limitante para las dietas balanceadas, en especial para cultivos intensivos de tilapias, así, se puede afirmar que los MV evaluados, teniendo en cuenta el CDA de la PB (Tabla 4) son adecuadas para su inclusión en la dieta de la tilapia nilótica, gracias a su alta digestibilidad y bajo contenido de fibra (Tabla 2). No obstante, que todos los CDA de la proteína bruta superan el $90 \%$, las diferencias estadísticas observadas entre los MV están dadas posiblemente por la calidad de la proteína en base seca de cada uno de los forrajes evaluados, y sea éste el principal factor que haya determinado la variabilidad de los resultados (Gutiérrez-Espinosa et al., 2011). 
El EE, en base seca, de los MV estudiados presentó valores entre 0,89 a 4,47\% (Tabla 3), cifras similares a las observadas en diferentes especies forrajeras, las cuales oscilan entre 1,4 a 6,5\% (Savón et al., 2006), con porcentajes de digestibilidad en la tilapia nilótica de 87,7 a 98,7\%. La diferencia de digestibilidad entre la fracción lipídica del parénquima de la hoja de los MV investigados, se puede deber a que cada uno de ellos contiene diferente composición y algunos factores antinutricionales, que limitan su absorción en el tracto gastrointestinal de los peces; así, en el Taro (Alocasia macrorrhiza) predominan las grasas neutras con $60,5 \%$, los glucolípidos $19,0 \%$, los fosfolípidos $20,5 \%$, los ácidos grasos linoléico y palmítico, aunque también están presentes el oleico, típico de los aceites vegetales, el linolénico, en el Bore (Xanthosoma sagitiffolium) otras sustancias químicas como carotenoides del grupo de los tetraterpenos entre ellos la beta-criptoxantina, los alfa-carotenos y beta-carotenos; al analizar la fracción grasa de las hojas matarratón (Gliricidia sepium) Kaniampady et al. (2007) encontraron sustancias como el propilenglicol (25,1\%), coumarin (18,2\%), (Z)-3-hexenol (17,7\%), $\beta$ farnesene $(14,2 \%)$ y hexonol $(6,5 \%)$. En el Nacedero (Trichantera gigantea), se reporta en el EE la presencia de factores anti-nutricionales como los taninos (Savón et al., 2002) que pueden disminuir la calidad nutritiva de la misma y finalmente, en los aceites del Botón de oro (Tithonia diversifolia) se han separado veinte terpenoides, siendo el principal constituyente el Z-beta ocimene, monoterpenoide responsable de dar el olor característico a la planta (Lamaty et al., 1991). Los resultados obtenidos de la digestibilidad del EE de la harina de los follajes evaluados en la tilapia nilótica durante la fase de crecimiento son altos y comparables con los de la harina de soya integral con fitasa (De Castro et al., 2005), el fríjol cargamanto (Phaseolus vulgaris) y la quinua (Chenopodium quinoa),, que presentan valores de CDA para la fracción lipídica de 95,73\%, 83,0\% y 67,9\%, respectivamente (WillsFranco et al., 2009). Las tilapias no utilizan eficientemente los lípidos como fuente energética en niveles por encima del $5 \%$ de la dieta, sin embargo, requieren en su dieta como nutrientes esenciales ácido linoléico y araquidónico para favorecer la ganancia de peso y la eficiencia alimenticia (Lee et al., 2002). Como se mencionó anteriormente, algunos follajes evaluados presentan alto nivel de ácido linoléico, característica que puede haber favorecido los altos CDA de su fracción grasa.

En el Botón de oro Gallego-Castro et al. (2014) determinaron el contenido de FDA en 34,48\%, en el Bore $20,56 \%$ y en el Nacedero valores entre 16,31\% y 41,66\%, Costa et al.(2009), al analizar el Matarratón, encontró contenidos de $24,30 \%$. En la presente investigación, la HN fue la que presentó el menor contenido de FDN en base seca con 4,06\% y al mismo tiempo la que obtuvo el mejor porcentaje de CDA de esta fracción con $64,7 \pm 2,7 \%$. La HB con $7,73 \%$ de FDA en base seca presentó el CDA más bajo de 18,5\% (Tabla 4). Al respecto, es importante resaltar que Lanna et al. (2004) observaron que niveles de fibra bruta en la dieta de la tilapia nilótica en crecimiento hasta del $5 \%$ no influyen en la digestibilidad de la materia seca ni de la proteína cruda. La variación en el contenido de FDA en los MV y su digestibilidad en la tilapia nilótica, puede estar influenciada por el tipo de cultivo, edad y tiempo de corte, uso de fertilizantes y las pruebas de laboratorio usadas para su determinación. El ELN incluye los carbohidratos más solubles: azúcares, almidón y algunas hemicelulosas, de un alto porcentaje de digestibilidad. La clase de carbohidrato determina su valor como fuente de energía para el animal. Se considera, además, que los cereales contienen del $60 \%$ al $70 \%$ de ELN, con bajo nivel de FB. Los valores obtenidos para los MV utilizados están en un rango entre 44,46\% y 58,45 (Tabla 2), para el CDA del ELN se obtuvo valores de 87 a $96,5 \%$ (Tabla 4), con excepción del HBO con 60,2\%, corroborando que a mayor valor del CDA de la FDN y de la FDA, el CDA del ELN se restringe debido a que la fibra causa disminución en el tiempo de tránsito del alimento por el tracto gastrointestinal (Pimenta et al., 2008).

Los contenidos de calcio y fósforo en base fresca de los MV investigados son bajos, solamente la HM presentó 1,45\% de $\mathrm{Ca}$ en base fresca y 7,01\% en base seca, aunque estos valores concuerdan con los valores descritos por Suárez et al., (2008). A excepción de la HB que presentó CDA de 72,2\% y 50,4\% para $\mathrm{P}$ y Ca, respectivamente, en las demás harinas los CDA de estos dos minerales superaron el $90 \%$. TorresNovoa y Hurtado-Nery (2012) sostienen que la relación Ca:P en los peces se debe mantener lo más próximo a uno, sin exceder la relación de 1,5:1, respectivamente, para disminuir los efectos contaminantes del medio ambiente. Para la tilapia la fuente de mayor disponibilidad de fósforo son las sales fosfato, ya que del $60 \%$ al $70 \%$ del fósforo de los vegetales se encuentra como fósforo fítico, lo que lo hace menos disponible para los no rumiantes. Sin embargo, al observar los CDA tanto del Ca como del P de las harinas del parénquima de los MV, superan el $70 \%$, se puede afirmar que la tilapia nilótica digiere eficientemente el $\mathrm{P}$ contenido en estos ingredientes, posiblemente, resultado de la adaptación de la especie al consumo de follajes. Los oxalatos de calcio presentes en el Bore son considerados como un factor antinutricional (Gomez, 2002) porque disminuyen la digestibilidad del alimento de los diferentes nutrientes. Los MV utilizados presentaron rangos para el CDA para el Ca entre un 94,1 y 95,4 \% con excepción de la HB que presenta un valor de 50,4\% (Tabla 4). Para el CDA del P los valores fluctuaron entre 72,2 y 93,7\% (Tabla 4). Se han evaluado materias primas alternativas en la alimentación del género Oreochromis donde la soya integral presenta del $30,1 \%$ al $53,7 \%$ y para la harina de trigo y el gluten de maíz valores de 46,6 y 47,9\%, respectivamente (Rueda-Uribe et al., 2012). Zhou y Yue (2012) al trabajar con harina de soya, harina de canola, harina de maní y semillas de algodón encontraron CDA para el fósforo entre 52,65 a 64,23\%. 
Los peces por ser animales poiquilotermos, tienen requerimientos de energía de mantenimiento inferiores a los de las especies homeotermas, además son más eficientes para digerir y metabolizar la proteína y los lípidos para obtener la mayor parte de la energía requerida por el organismo (Torres-Novoa y Hurtado-Nery, 2012). Los MV en base seca presentaron una EB entre 3112,51 y 4348,77 Kcal/kg (Tabla 2), con CDA para $\mathrm{HBO}, \mathrm{HB}, \mathrm{HN}$ y $\mathrm{HM}$ de $57,3 \%, 78,4 \%, 73,5 \%$ y $75,2 \%$, respectivamente, resultados que corroboran la afirmación anterior. Igualmente, se resalta que en la presentación de harina del parénquima de la hoja, fueron la $\mathrm{HBO}$ y $\mathrm{HB}$ las que presentaron el mayor contenido de energía digestible por kilogramo de ingrediente, posiblemente gracias a sus altos contenidos de PB y EE, respectivamente.

En general los CDA de los nutrientes de los MV estudiados en esta investigación para PB, EE, Ca, P, carbohidratos solubles y EB son altos, a excepción de dos valores, superan el 70\%. Además, se demostró que la tilapia nilótica tiene capacidad de digerir parcialmente la FDA, aunque no tan eficientemente como un rumiante. Como ventaja adicional los resultados muestran ingredientes con potencial de uso al ser poco agresivos con el medio ambiente, ya que los MV que poseen un CD mayor del $50 \%$ no causan grandes pérdidas por eliminación de nutrientes en la materia fecal reduciendo con esto su impacto negativo en el medio acuático (Pimenta et al., 2008).

Las diferencias estadísticas observadas al comparar las medias de los CD entre plantas dentro de un mismo nutriente $(p<0,05)$, se pueden deber a factores externos correlacionados a características del material vegetal evaluado (Vásquez-Torres et al., 2010), ya que, la metodología utilizada para los diferentes procesos experimentales, análisis de las dietas, condiciones medio ambientales atmosféricas y acuáticas, especie y edad de los peces fueron similares entre tratamientos.

\section{CONCLUSIONES}

Se concluye que, la harina del parénquima de las hojas de los Materiales Vegetales evaluados: Botón de oro (Tithonia diversifolia) (Hemsl.) A. Gray, Bore (Xanthosoma sagittifolium (L) Schott, Nacedero (Trichanthera gigantea) (Bonpl.) Nees y Matarratón (Gliricidia sepium) (Jacq.) Kunth ex Walp, suministradas a ejemplares machos juveniles de tilapia nilótica (Oreochromis niloticus, Trewav. 1984), son eficientemente digeridos por estos, sugiriendo así que estas materias primas, tanto en base seca como en base fresca, son ingredientes alternativos valiosos en la alimentación de esta especie, en especial para sistemas de piscicultura de subsistencia, donde se disponga de estos recursos vegetales, permitiendo disminuir los costos de producción y el impacto ambiental en el medio acuático.

\section{REFERENCIA}

Association of Official Analytical Chemists-AOAC, Official methods of analysis. 16th ed., Ed. S. Willians, Arlington, Virginia, USA (1995)

Brenes-Soto, A. Respuesta productiva de conejos alimentados con follaje fresco de nacedero (Trichanthera gigantea, Lamiales: Acanthaceae), Cuadernos de Investigación UNED, 6(2), 205-211 (2014)

Costa, B.M., da, Santos, I.C.V., Oliveira, G.J.C. e I.G de Pereira. Avaliação de folhas de gliricidia sepium (JACQ.) walp por ovinos. Arch. Zootec, 58(221), 33-41 (2009)

De Castro S., T.S., W.M. Furuya, y otros 7 autores, Coeficientes de digestibilidade aparente da energia e nutrientes do farelo de soja integral sem e com fitase para a tilápia do Nilo (Oreochromis niloticus), Acta Sci. Anim. Sci., 27(3), 371-376 (2005)

El-Saidy, D.M.S.D. y A.S. Saad. Evaluation of Cow Pea Seed Meal, Vignasinensis, as a Dietary Protein Replacer for Nile tilapia, Oreochromis niloticus (L.), Fingerlings, Journal of the World Aquaculture Society, 39, 636-645 (2008)

Forster, I., A note on the method of calculating digestibility coefficients of nutrient provided by single ingredients to feeds of aquatic animals, Aquaculture Nutrition, 5, 143-145 (1999)

Gallego-Castro, L. A., L. Mahecha-Ledesma y J. Angulo-Arizala, Potential of Tithonia diversifolia Hemsl. A Gray as forage for the production of dairy cows, Agron. Mesoam, 25 (2), 393-403 (2014)

Garduño-Lugo, M. y M.A. Olvera-Novoa, Potential of the use of peanut (Arachis hypogaea) leaf meal as a partial replacement for fish meal in diets for Nile tilapia (Oreochromis niloticus L.), Aquaculture Research, 39, 1299-1306 (2008)

Giraldo, A., Velasco, R.J. y H.S. Villada, Digestibilidad Aparente de una Harina Proveniente de Hojas de Yuca (Manihot esculenta, Crantz), Información Tecnológica, 19(1), 11-18 (2008) 
Gómez Z., M.E., Bore Alocasia macrorrhiza (Linneo.) Schott, una especie con Potencial para la Agroforestería PecuariaTres especies vegetales promisorias: Nacedero Trichathera gigantea (H. \& B.) Nees; Botón de oro Tithonia diversifolia (Hemsl) Gray; Bore Alocasia macrorrhiza (Linneo) Schott. Editores: Sonia D. Ospina, Enrique Murgueitio. CIPAV. Cali, Valle del Cauca, Colombia, 13-23, 301p (2002)

González-Castillo, J. C., Hahn von-Hessberg, C. M. y Narváez-Solarte, W., Características botánicas de Tithonia diversifolia (Asterales: Asteraceae) y su uso en la alimanetación animal, Bol.Cient.Mus.Hist.Nat. 18 (2), 45-58 (2014)

Gutiérrez-Espinosa, M., M. Yossa-Perdomo y W. Vásquez-Torres, Digestibilidad aparente de materia seca, proteína y energía de harina de vísceras de pollo, quinua y harina de pescado en tilapia nilótica, Oreochromis niloticus, Orinoquia, 15(2), 169-179 (2011)

Hahn Von-H, C.M., A. Grajales Q., J.E. Vargas y J. Muñoz, Alimentación de Tilapia (Oreochromis niloticus) con follaje de Morera (Morus sp.) y Bore (Alocasia macrorrhiza (Linneo), Schott.), In ColombiaTres Especies Vegetales Promisorias, Nacedero (Trichanthera gigantea) (H.\&B.) Nees; Botón de Oro (Thitonia diversifolia) (Hemsl.) Gray y Bore (Alocasia macrorrhiza) (Linneo), CIPAV, 49-64, Cali, Valle del Cauca, Colombia (2002)

Kaniampady, M., M. Muhammed, L. Jirovetz y P. Mohamed, Essential composition of Gliricidia sepium (Leguminosae) leaves and flowers, Indian J. Chem, 46B, 1359-1360, (2007)

Kavanagh, S., P.B. Lynch, F. O'mara, y P.J. Caffrey, A comparison of total collection and marker technique for the measurement of apparent digestibility of diets for growing pigs, Animal feed science and technology, 89(1), 49- 58 (2001)

Lamaty, G., C. Menut, P.H. Zollo, J.R. Kuiate, J.M. Bessiere y J. Kodou, Aromatic plants of tropical central Africa. III. Contituents of the essential oil of the leaves of Tithonia diversifolia (Hemsl.) Gray from Cameroon, Journal of Essential oil Research, 3(6), 399-402 (1991)

Lanna, E.A., L.E. Pezzato, P.R. Cecon, W.M. Furuya, M.A. y Bomfim, Digestibilidade Aparente e Trânsito Gastrintestinal em Tilápia do Nilo (Oreochromis niloticus), em Função da Fibra Bruta da Dieta, Revista Brasileira de Zootecnia, 33, 2186-2192 (2004)

Lee, S.M., I.G. Jeon, J.Y. Lee, Effects of digestible protein and lipid levels in practical diets on growth, protein utilization and body composition of juvenile rockfish (Sebastes schlegeli), Aquaculture, 211, 227-239 (2002)

Narváez, W., D.I. Hurtado y S. Nocua, Valor nutricional de la morera (Morus sp.), matarratón (Gliricidia sepium), pasto india (Panicum máximum) y arboloco (Montanoa quadrangularis) en la alimentación de cuyes (Cavia porcellus), Vet. Zootec, 6(1), 56-65 (2012)

National Research Council, NRC. Nutrient requirements of warm water fishes and shellfishes, National Academy Press, Washington, D.C., USA (1993)

Nhi, N.H.Y. y T.R. Preston, Growth of red tilapia (Oreochomis mossambicus) and black tilapia (Oreochomis niloticus) with common carp (Ciprinus carpio) in monoculture and polyculture by using natural feed in ponds fertilized with biodigester effluent, Livestock Research for Rural Development, 24(111) (2012)

Pimenta, M.E.S, M.M. Oliveira, P.V.R. Logato, C.J. Pimenta y T.A. Freato, Desempenho produtivo e digestibilidade pela tilápia do nilo (Oreochromis niloticus, Linnaeus, 1758) alimentada com dietas suplementadas com níveis crescentes de silagem ácida de pescado, Ciênc Agrotec, 32, 1953-1959 (2008)

Rivas-Vega, M.E., J.L. López-Pereira, A. Miranda-Baeza y M.I. Sandoval-Muy, Sustitución Parcial de Harina de Sardina con Moringa oleifera en Alimentos Balanceados para Juveniles de Tilapia (Oreochromis mossambicus $x$ Oreochromis niloticus) cultivada en agua de mar, Revista de Ciencias Biológicas y de la Salud, 2, 3-10 (2012)

Rodríguez, N., E.O. Simões y R. Guimarães-Junior, Uso de indicadores para estimar consumo y digestibilidad de pasto. Lipe, lignina purificada y enriquecida Revista Colombiana de Ciencias Pecuarias, 20(4) (2007)

Rueda-Uribe, W., W. Vásquez-Torres y M. Gutiérrez-Espinosa, Digestibilidad de fósforo y proteína de raciones suplementadas con fitasa en tilapia, Oreochromis sp. Orinoquia, 16(1) (2012)

SAS Institute. JMP Statistics and Graphic Guide. Version 7.0 SAS Institute Inc., Cary, NC. (2007) 
Savón L, I. Scull, M. Orta y V. Torres, Caracterización físicoquimica de cinco harinas de follajes tropicales para especies monogástricas, Revista Cubana de Ciencias Agrícolas, 38(3), 291-296 (2002)

Savón, L., L.E. Dihigo, I. Scull, O. Gutiérrez, A. Albert y M. Orta, Valor Nutritivo del Follaje de Tricantera (Trichanthera gigantea) en Animales Monogástricos, Revista Computadorizada de Producción Porcina, 13(1), 79 (2006)

Secombe, C.J. y G.D. Lester, The role of diet in the prevention and management of several equine diseases, Animal feed science and technology, 173(1-2), 86-101 (2012)

Sklan, D., T. Prag y I. Lupatsch, Apparent digestibility coefficients of feed ingredients and their prediction in diets for tilapia Oreochromis niloticus x Oreochromis aureus (Teleostei, Cichlidae), Aquaculture Research, 35, 358-364, (2004)

Suárez S., J.C., J.E. Carulla y J.E. Velásquez, Composición química y digestibilidad in vitro de algunas especies arbóreas establecidas en el piedemonte Amazónic Centro Agronómico Tropical de Investigación y Enseñanza. Turrialba, Costa Rica. Revista Zootecnia Tropical. 26(3) (2008)

Torres-Novoa, D.M. y V.L. Hurtado-Nery, Requerimientos nutricionales para Tilapia del Nilo (Oreochromis niloticus), Orinoquia, 16(1) (2012)

Vásquez-Torres, W., M.I. Yossa, G. Hernández y M.C. Gutiérrez, Digestibilidad aparente de ingredientes de uso común en la fabricación de raciones balanceadas para tilapia roja híbrida (Oreochromis sp), Revista Colombiana de Ciencias Pecuarias, 207-216 (2010)

Vásquez-Torres, W., M.Y. Yossa y M.C. Gutierrez Espinosa, Digestibilidad aparente de ingredientes de origen vegetal y animal en la cachama, Pesq. agropec. bras, 48(8), 920-927 (2013)

Van Soest, P. J., Robertson, J. D. y B. A. Lewis, Methods for dietary fiber, neutral detergent fiber, nonstarch polysaccharides in relation to animal nutrition. Journal of Dairy Science. Chanpaign, 74, 3583-3597 (1991)

Verdecia, D.M., J. Ramírez, I. Leonard, Y. Álvarez, Y. Bazán, R. Bodas, S. Andrés, J. Álvarez, F. Giráldez y S. Lopez, Calidad de la Tithonia diversifolia en una zona del Valle del Cauto, Revista electrónica de Veterinaria, 12(5) (2011)

Wills-Franco, G.W., A.P. Muñoz-Ramirez y A.M. Valderrama-Gama, Determinación de coeficientes de digestibilidad aparente de materias primas de origen orgánico para alimentación de tilapia roja (Oreochromis sp), Rev Colomb Cienc Pecu, 22(3) (2009)

Zhou, Q.-C. y Y.R. Yue, Apparent digestibility coefficients of selected feed ingredients for juvenile hybrid tilapia, Oreochromis niloticus × Oreochromis aureus, Aquaculture Research, 43, 806-814 (2012) 